\title{
ABCG1 maintains high-grade glioma survival in vitro and in vivo
}

\author{
Yi-Hsien Chen ${ }^{1}$, Patrick J. Cimino², Jingqin Luo ${ }^{3}$, Sonika Dahiya ${ }^{2}$, David H. Gutmann ${ }^{1}$ \\ ${ }^{1}$ Department of Neurology, Washington University School of Medicine, St. Louis, MO, USA \\ ${ }^{2}$ Department of Pathology, Washington University School of Medicine, St. Louis, MO, USA \\ ${ }^{3}$ Department of Surgery, Washington University School of Medicine, St. Louis, MO, USA
}

Correspondence to: David H. Gutmann, e-mail: gutmannd@neuro.wustl.edu

Keywords: glioblastoma, ER stress, brain tumor, glioma stem cell, apoptosis

Received: September 29, $2015 \quad$ Accepted: February 25, $2016 \quad$ Published: March 10, 2016

\section{ABSTRACT}

The overall survival for adults with malignant glioma (glioblastoma) remains poor despite advances in radiation and chemotherapy. One of the mechanisms by which cancer cells develop relative resistance to treatment is through de-regulation of endoplasmic reticulum (ER) homeostasis. We have recently shown that ABCG1, an ATP-binding cassette transporter, maintains ER homeostasis and suppresses ER stress-induced apoptosis in low-grade glioma. Herein, we demonstrate that ABCG1 expression is increased in human adult glioblastoma, where it correlates with poor survival in individuals with the mesenchymal subtype. Leveraging a mouse model of mesenchymal glioblastoma (NPcis), shRNA-mediated Abcg1 knockdown (KD) increased CHOP ER stress protein expression and resulted in greater NPcis glioma cell death in vitro. Moreover, Abcg1 KD reduced NPcis glioma growth and increased mouse survival in vivo. Collectively, these results demonstrate that ABCG1 is critical for malignant glioma cell survival, and might serve as a future therapeutic target for these deadly brain cancers.

\section{INTRODUCTION}

Brain tumors represent the fourth leading cause of cancer-related death in adults, where high-grade glial neoplasms (malignant gliomas) predominate [1]. These malignant gliomas are classified by the World Health Organization (WHO) as grade III (anaplastic astrocytoma, anaplastic oligodendroglioma, and anaplastic mixed oligoastrocytoma) or IV (glioblastoma) astrocytomas, commonly occurring in individuals between the ages of 45 and 75 years [2]. Unfortunately, survival following the diagnosis of a glioblastoma is dismal, with most patients dying within 12-16 months despite aggressive surgical, radiation, and chemotherapy management $[1,3]$. Since these cancers are thought to be maintained by cells with stem cell-like properties (glioma stem cells; GSCs), intense focus over the past decade has centered on understanding the critical pathways that govern GSC growth and differentiation $[4,5]$.

In this regard, a large number of studies have revealed that GSCs are capable of long-term self-renewal, multi-lineage differentiation, and the generation of histologically-similar tumors following implantation into immunocompromised rodent brains [6]. Most often identified by their expression of the prominin-1 cell surface marker, CD133 [6, 7], GSCs can be enriched by antibody-mediated isolation, and shown to possess unique properties such as radioresistance and chemoresistance not shared with normal brain neural stem cells (NSCs) [8-10]. Another of these markers is the ABCG2 protein, which identifies the "side population", a subset of stem cells hypothesized to be enriched for cancer propagation abilities $[9,11]$.

Similar to ABCG2, ABCG1 belongs to a large family of ATP-binding cassette proteins involved in cellular transport $[12,13]$, where it mainly directs lipid transport $[14,15]$. In this regard, we recently leveraged a low-grade glioma mouse model of Neurofibromatosis type 1 (NF1)-associated optic glioma to identify and characterize low-grade glioma (LGG) cancer stem cells (LG-GSCs) [16]. Similar to their malignant glioma counterparts, these LG-GSCs were capable of long-term self-renewal, multi-lineage differentiation, and forming low-grade glioma-like lesions following explantation into immunocompetent mice. However, ABCG2 was not highly expressed in these murine LG-GSCs, consistent with an absence of a side population following Hoechst 33342 flow cytometry. Instead, ABCG1 was highly 
enriched in both murine and human LG-GSCs relative to their non-neoplastic NSC counterparts, where it served to protect these cells from ER stress-mediated cell death (apoptosis).

Based on the controversial use of ABCG2 as a marker of CSCs [11] and the recent identification of ABCG1 as a critical mediator of LG-GSC survival, we sought to determine whether ABCG1 was important for high-grade (glioblastoma) survival. In this report, we demonstrate that $A B C G 1$ expression negatively correlates with survival in patients with glioblastoma of the mesenchymal subtype. Leveraging a mouse model of mesenchymal glioblastoma (NPcis), characterized by Nfl and Trp53 loss, we show that shRNA-mediated Abcgl knockdown increases ER stress-induced apoptosis in vitro as well as improves the survival of immunocompetent mice with glioblastoma in vivo. Collectively, these results establish ABCG1 as another potential biomarker for high-grade glioma survival relevant to future brain tumor therapeutic targeting.

\section{RESULTS}

\section{Glioblastoma ABCG1 expression is associated with reduced patient survival}

We have recently shown that human low-grade (pilocytic astrocytoma) glioma specimens exhibit increased ABCG1 expression [16]. To determine whether ABCG1 expression was similarly increased in human glioblastoma specimens, two sets of experiments were performed. Using the one available GEO dataset containing normal reference tissue (astrocytes; GSE15824), we found that $A B C G 1$ expression was increased in glioblastoma tumors $(697 \pm 554,230913$ at probe set; $428 \pm 305,232081 \_$at probe set) relative to astrocytes $(11.5 \pm 1.1,230913$ _at probe set; $13.9 \pm 8.1$, 232081_at probe set). Similarly, increased ABCG1 immunoreactivity was observed in representative tumor specimens from human patients with glioblastoma $(n=16)$ relative to normal brain controls $(n=7)$ (Figure $1 \mathrm{~A}$ and S1).

Next, we sought to determine whether $A B C G 1$ expression was associated with clinical outcome (overall survival) in patients with high-grade glioma (GSE16011), the majority of whom did not receive prior chemotherapy. Using a total of 159 glioblastoma patients dichotomized by the median of $A B C G 1$ expression, there were no significant survival differences between patients harboring gliomas with low versus high $A B C G 1$ gene expression (Figure 1B). However, when the analysis was stratified by glioblastoma molecular subtype, high $A B C G 1$ expression correlated with shorter overall survival only in the mesenchymal subgroup (Figure 1C), a subtype characterized by $N F 1$ gene mutation [17]. Moreover, using multivariate Cox hazard regression analysis with adjustment for both age and Karnofsky Performance Score (KPS), the negative prognostic value of $A B C G 1$ expression in mesenchymal GBM patient survival remained significant $(P=0.017$; Figure 1D). Together, these findings demonstrate increased ABCG1 expression in human malignant gliomas, where it is associated with reduced survival in patients harboring the mesenchymal glioblastoma subtype.

\section{Abcg1 knockdown decreases glioblastoma cell growth in vitro}

In the glioblastoma mesenchymal molecular subtype, NF1 loss is frequently associated with TP53 mutation [17]. Consistent with this observation, mice harboring germline inactivating mutations in both the Nf1 and Trp53 genes residing on the same chromosome (NPcis mice) spontaneously develop high-grade gliomas $[18,19]$. Moreover, derivative glioma cells from these NPcis mouse tumors exhibit bi-allelic loss of the Nf1 and Trp53 genes in addition to increased PDGFR $\alpha$ expression, molecular features also observed in human mesenchymal glioblastomas [17, 20, 21]. Given the correlation between $A B C G 1$ expression and overall survival in patients with mesenchymal subtype glioblastoma, we sought to determine whether ABCG1 might be important for NPcis glioma growth using lentivirus-mediated shRNA knockdown in two independently-generated NPcis highgrade glioma cell lines (K1861 and K4622; [22]). First, we confirmed Abcg1 knockdown using two distinct shRNA constructs by Western blotting (Figure 2A; 50\% protein reduction). Following Abcg1 knockdown, K1861 cells exhibited morphological changes (e.g., rounding) suggestive of cell death (Figure 2B). Consistent with these cellular changes, reduced cell growth $\left(\% \mathrm{Ki}^{+} 7^{+}\right.$ cells; Figure 2C) and increased apoptosis was observed ( $\%$ cleaved caspase- $3^{+}$cells; Figure $2 \mathrm{C}$ ) relative to shRNA controls. Identical results were also observed using the K4622 high-grade glioma cell line (Figure S2).

\section{Abcg1 knockdown increases ER stress and glioblastoma cell apoptosis}

Since Abcg1 confers a survival advantage to LGGSCs by suppressing ER stress [16], we examined the expression of BiP and CHOP, two markers of ER stress. Following Abcg1 knockdown (45-54\% protein reduction) in K1861 and K4622 glioblastoma cells, there was increased CHOP expression (2.3 and 3-fold, respectively) and caspase- 3 cleavage (1.5 and 2.2-fold, respectively) relative to controls (Figure 3 ). However, in contrast to LG-GSCs, there was no change in BiP expression upon Abcg1 reduction. 


\section{Abcg1 knockdown reduces glioma growth and mouse survival in vivo}

To determine whether Abcg1 knockdown also decreases glioma growth in vivo, $5.0 \times 10^{4} \mathrm{~K} 1861$ glioblastoma cells were stereotactically injected in the right striatum of 3-week-old C57B1/6J mice. Since these K1861 cells were transduced with a lentiviral vector expressing green fluorescent protein and firefly luciferase, tumor growth could be monitored by live bioluminescence imaging (BLI). We specifically chose to employ the K1861 glioblastoma for these in vivo experiments, based on previous studies demonstrating that K1861-engrafted mice develop large and aggressive intracranial tumors $[19,23]$. Whereas all engrafted mice harbored brain tumors, there was reduced tumor growth by BLI in the two K1861 Abcg1 shRNA groups ( $n=10$ mice/group) relative to control shRNA K1861-implanted mice $(n=10$ mice $)$ at 28 days post-injection (Figure 4A) and the bioluminescent signal was significantly reduced in both $A b c g 1$ shRNA groups relative to control shRNA K1861-implanted mice (\#1, $p=0.042 ; \# 2, p=0.0098$; Figure 4B). Over the 120 days of observation, mice in the shRNA control group became ill and had to be euthanized (median survival $=37$ days). However, in striking contrast, mice receiving Abcg1-shRNA K1861 glioblastoma cells had median survivals of $64(P<0.0001)$ and $98(P<0.0001)$ days, respectively (Figure 4C). While sh $A b c g 1-\# 2$ was slightly more effective at reducing Abcg1 protein expression than $\operatorname{sh} A b c g 1-\# 1$ ( $15 \%$ versus $30 \%$ of $\operatorname{sh} L a c Z$ levels), which might account for the observed lower BLI signal observed in mice receiving 1861 cells with shAbcg1-\#2 versus shAbcg1-\#1 $(P=0.0226)$, there was no difference in survival $(P=0.1426)$.

To determine whether Abcg1 knockdown resulted in increased glioblastoma apoptosis in vivo, $\mathrm{Ki} 67$ and TUNEL immunostaining were performed on the tumors following euthanasia. Consistent with the above in vitro findings, shAbcg1 K1861 tumors had reduced Abcg1 protein expression and fewer $\mathrm{Ki}^{+} 7^{+}$cells relative to
A

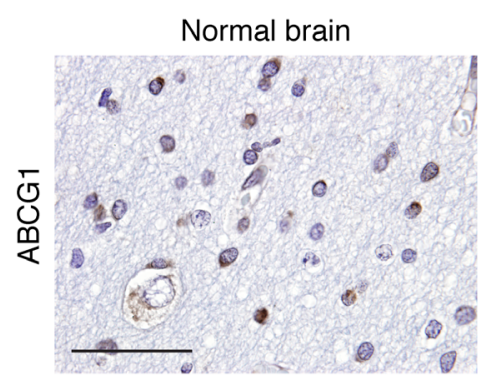

C

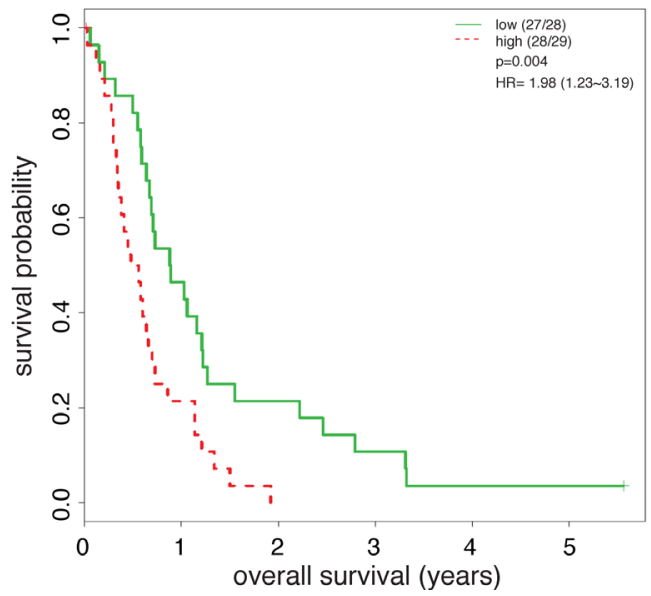

B

\begin{tabular}{ccc}
\hline Patients & HR (ABCG1: high vs. low) and \\
$95 \% \mathrm{Cl}$ & $\begin{array}{c}\text { log rank test } \\
\text { P-value }\end{array}$ \\
All & $1.15(0.89 \sim 2.29)$ & 0.342 \\
Mesenchymal & $1.98(1.23 \sim 3.19)$ & 0.004 \\
Proneural & $1.19(0.74 \sim 1.9)$ & 0.164 \\
Neural & $1.04(0.44 \sim 2.43)$ & 0.71 \\
Classical & $0.82(0.43 \sim 1.54)$ & 0.375 \\
\hline
\end{tabular}

$\mathrm{D}$

Figure 1: Abcg1 expression is associated with poor overall survival in the mesenchymal glioblastoma molecular subtype. (A) A representative specimen from one adult with a glioblastoma reveals increased ABCG1 expression relative to one representative agematched control brain. Scale bar, $50 \mu \mathrm{m}$. (B) Prognostic effect of ABCG1 (high vs. low) in all patients and within each subtype. (C) KaplanMeier analysis demonstrates that increased $A B C G 1$ expression negatively correlated with overall survival in patients with the mesenchymal glioblastoma subtype $(p=0.004)$ in GSE16011. (D) In GSE16011, while both age $(P=0.0023)$ and KPS $(P=0.0037)$ are associated with overall survival, multivariate analysis shows that high $A B C G 1$ expression is a strong predictor of poor patient survival, independent of age and $\operatorname{KPS}(P=0.017)$. 
A

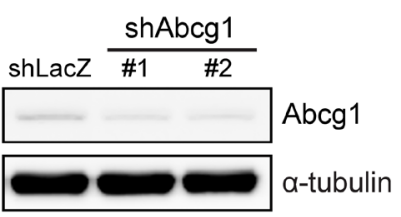

C
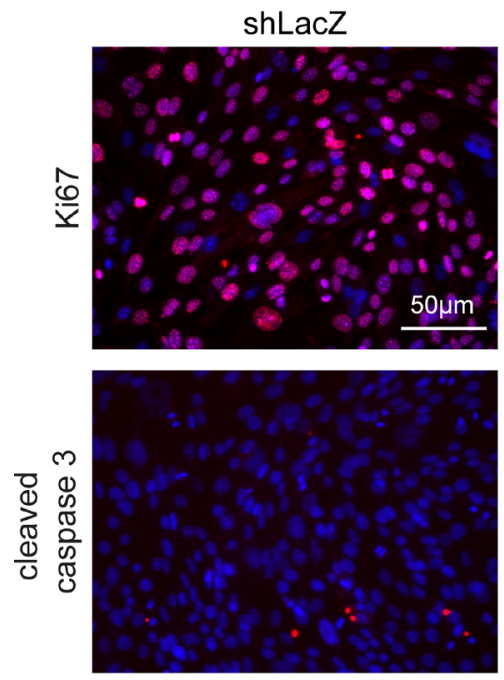

B
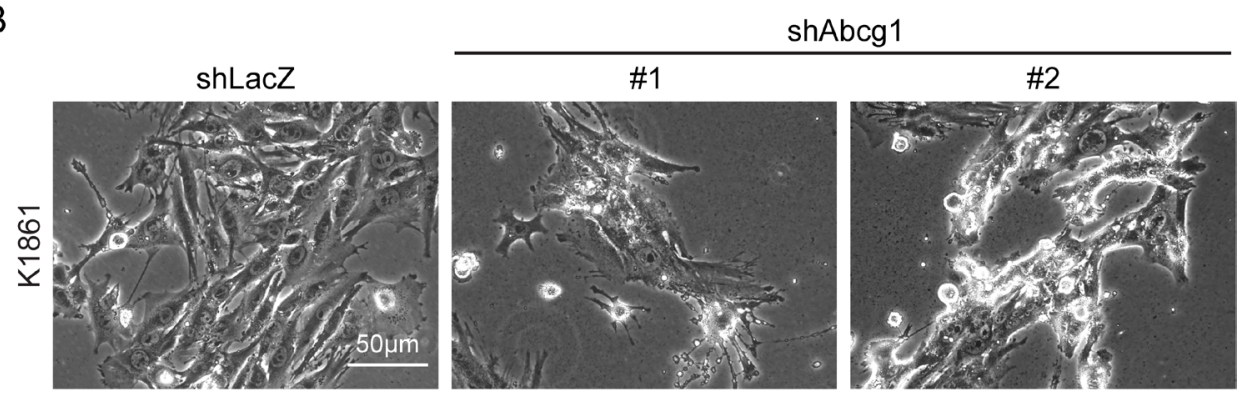

$\operatorname{shAbcg1}$
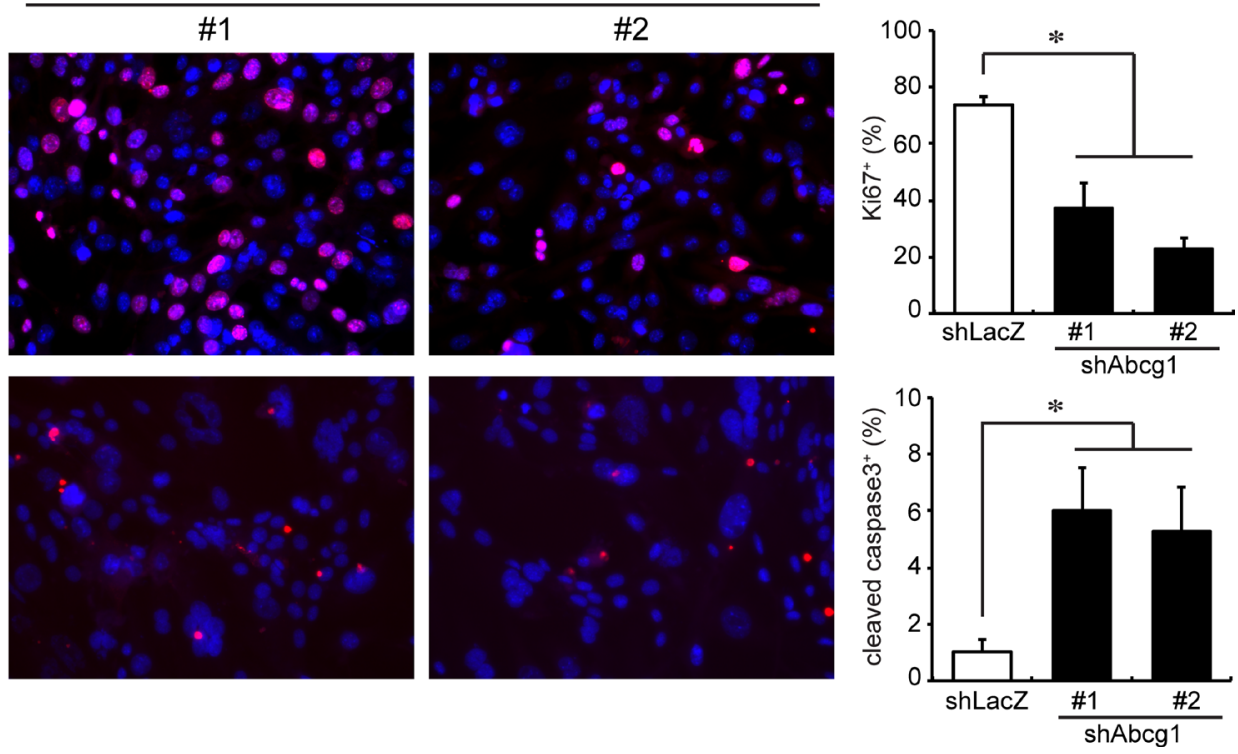

Figure 2: Abcg1 knockdown reduces NPcis glioma cell growth in vitro. (A) shRNA-mediated Abcg1 knockdown in K1861 NPcis glioma cells. (B) NPcis cells 6 days post-infection with sh $A b c g 1$ or control shLacZ virus. Following Abcg1 knockdown, there was (C) reduced cell growth ( $\%$ Ki6 $7^{+}$cells) and increased apoptosis ( $\%$ cleaved caspase $3^{+}$cells). Scale bar, $50 \mu \mathrm{m}$. Error bars denote mean $\pm \mathrm{SD}$. $(*) p<0.05$.

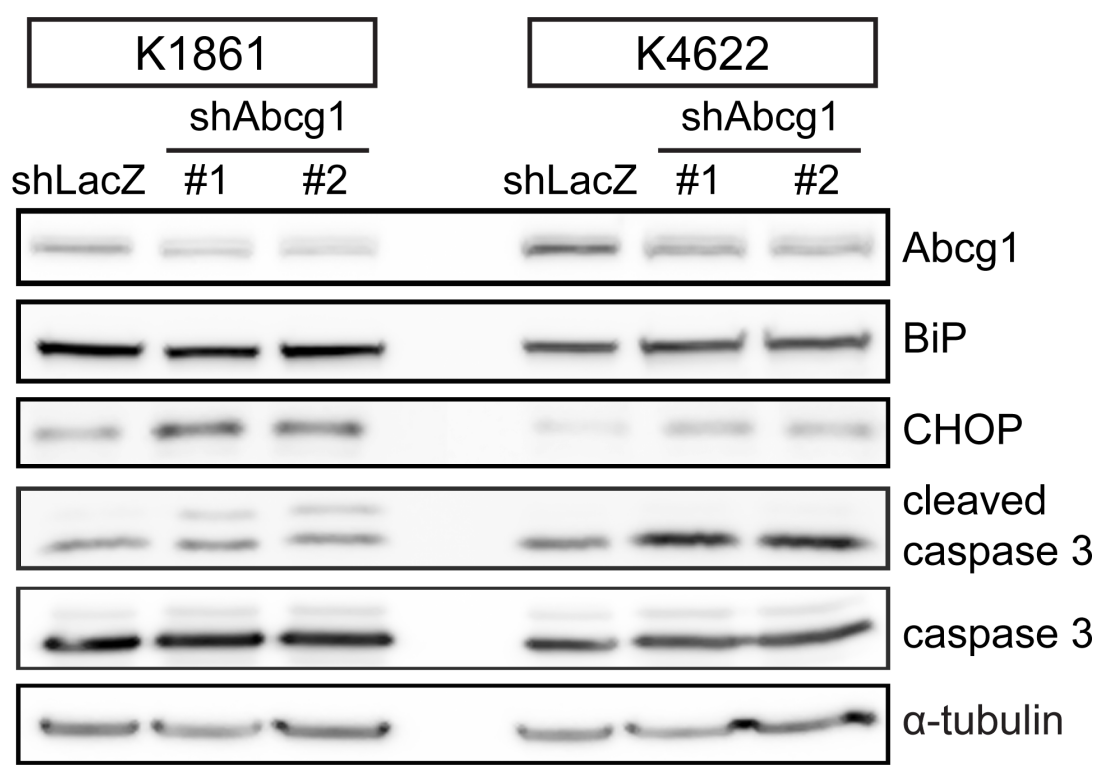

Figure 3: Abcg1 knockdown increases the ER stress response. shRNA-mediated Abcg1 knockdown in K1861 and K4622 glioblastoma cells increased CHOP and cleaved caspase-3, but not BiP, expression. 
controls (Figure 5). In addition, shAbcg1 K1861 tumors had increased cell death (TUNEL ${ }^{+}$cells). Collectively, these findings establish Abcg1 as a mediator of ER stressmediated apoptosis and survival in high-grade glioma.

\section{DISCUSSION}

Adaption to ER stress represents one protective mechanism for sustaining tumor cell growth and survival in the setting of hypoxia, low glucose levels, or chemotherapeutic drug exposure $[24,25]$. While little is known about ABCG1 in cancer, we demonstrate for the first time that ABCG1 is expressed in both murine and human glioblastoma, where its expression correlates with overall patient survival. Interestingly, $A B C G 1$ expression was not associated with survival in patients with all molecular subtypes of glioblastoma; however, there was a strong correlation between poor patient survival and high $A B C G 1$ RNA expression in tumors classified in the mesenchymal GBM subtype. In this subtype, one of the signature genetic changes is mutation of the NF1 tumor suppressor gene [17], which is intriguing, given the prior identification of ABCG1 as a uniquely upregulated transcript in GSCs originating from a mouse model of low-grade glioma harboring biallelic $N f 1$ gene inactivation $[16,26]$. Importantly, increased Abcg 1 expression does not

\section{A}
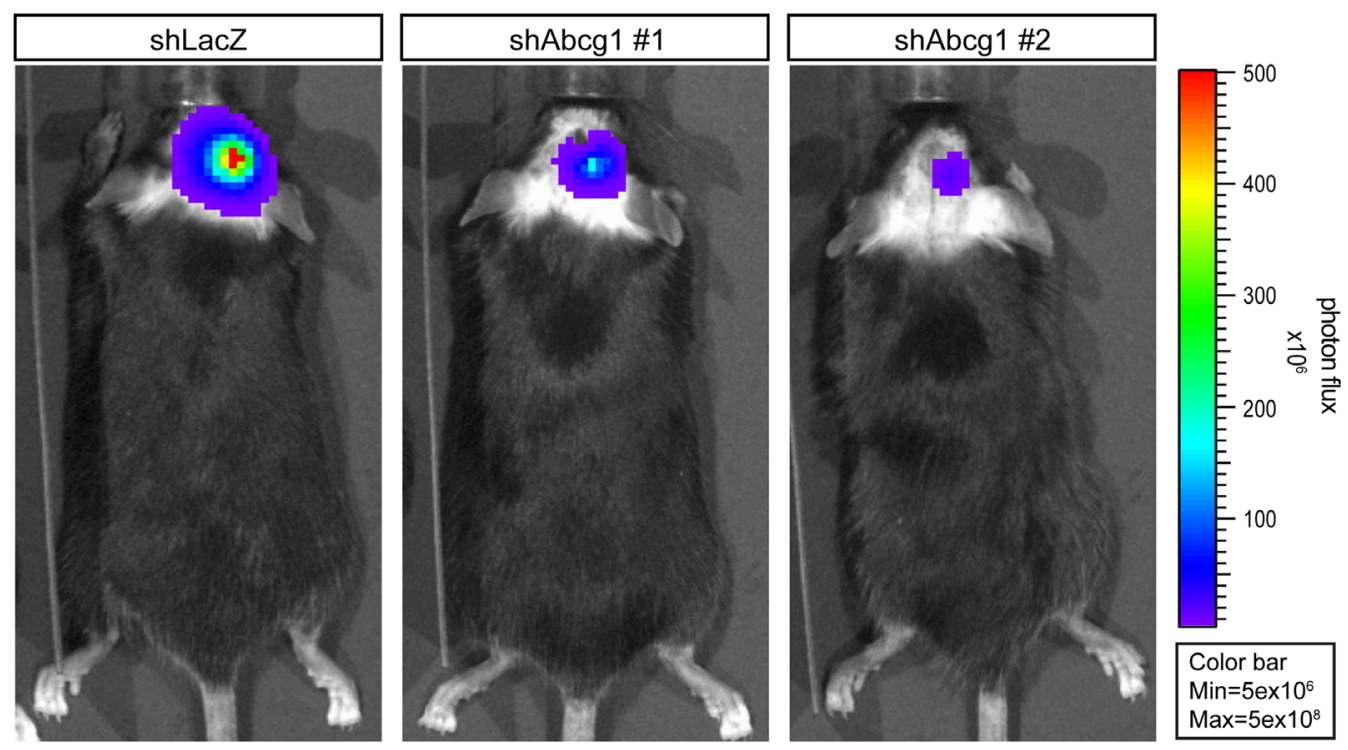

B

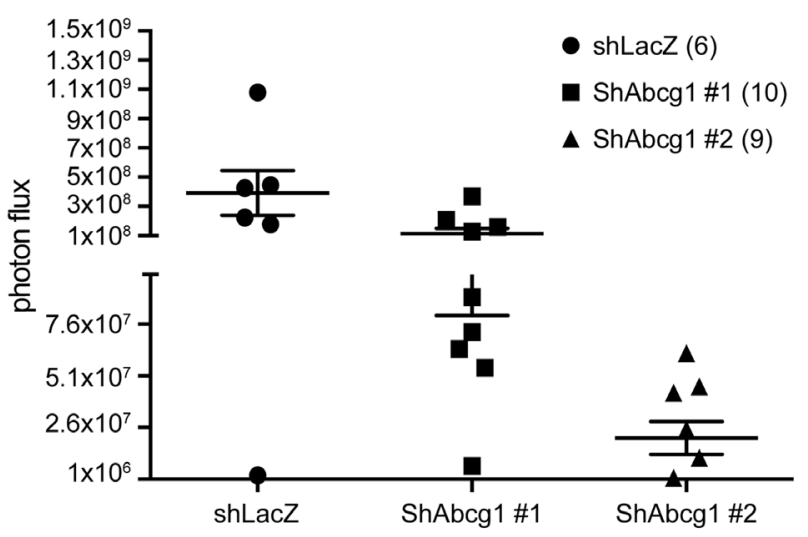

C

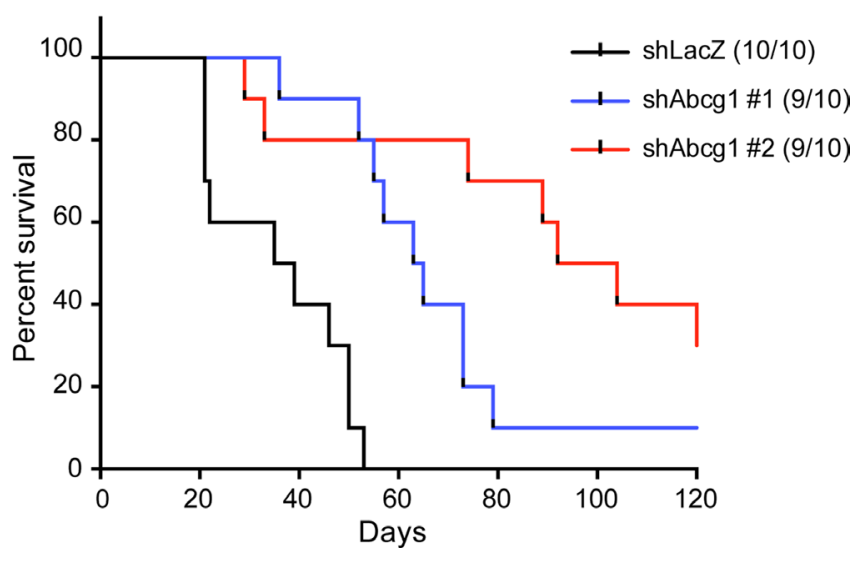

Figure 4: Abcg1 knockdown reduces tumor growth in vivo. (A) Representative bioluminescence images of tumors in engrafted mice from control $\operatorname{sh} L a c Z$ and $\operatorname{sh} A b c g 1$ groups (\#1, \#2) at 28 day post-transplantation were shown. (B) Photon flux of alive mice in all groups at 4 weeks post-transplantation. Relative to shLacZ-K1861 implanted mice, C57BL/6 mice receiving shAbcg1-K1861 tumors had reduced bioluminescence signal (\#1, $p=0.042$; \#2, $p=0.0098$ ). Error bars denote mean $\pm \mathrm{SEM}$. (C) Kaplan-Meier survival analysis reveals that mice transplanted with shAbcg1 K1861 NPcis cells exhibit longer survival than those injected with control (shLacZ) NPcis cells (log-rank test; $p<0.0001)$. 
result from $N f 1$ loss directly, but is rather a consequence of GSC development. In this regard, only optic glioma stem cells, but not Nfl-deficient NSCs, exhibit increased Abcg1 expression [16]. While it is currently not known how ABCG1 expression is regulated, a recent study conducted in $\mathrm{CHO}$ cells implicated E3-ubiquitin ligases (HUWE1 and NEDD4-1) in the post-translational regulation of ABCG1 protein stability and cholesterol export [27]. Formal demonstration of an ubiquitin ligase pathway that coordinately regulates neurofibromin and ABCG1 expression in glioma warrants further investigation [28, 29].

Similar to our findings in LG-GSCs, reduced Abcg1 protein expression using two different shRNA targeting constructs in two independently-generated NPcis glioblastoma lines (K1861 and K4622) increased the expression of CHOP, a final effector of the ER stress response, and resulted in higher levels of tumor cell death by apoptosis. However, in contrast to optic glioma stem cells, some molecular elements of the ER stress pathway were not conserved in glioblastoma cells. In this regard, we did not observe any changes in BiP expression, PDI expression, or PERK phosphorylation (data not shown), suggesting that other ABCG1-dependent mechanisms for inducing ER stress may be operative. Further studies focused on additional ER stress pathways in glioblastoma are currently being explored.

The fact that attenuated ABCG1 expression significantly prolongs mouse survival following glioblastoma implantation supports a critical role for this protein in tumor maintenance. This in vivo effect was demonstrated both in terms of tumor growth (bioluminescence imaging and tumor proliferation) and apoptosis (TUNEL), reminiscent of reported effects of ABCG2 in high-grade brain tumors. In these studies, $\mathrm{ABCG} 2$ has been implicated in reduced brain penetration of enhancer of zeste homolog 2 (EZH2) inhibitors [30] as well as the anti-tumoral responses to PARP inhibitors (ABT-888) and temozolomide [31]. Moreover, ABCG2 downregulation inhibits glioma stem cell migration and invasion [32, 33], where its expression partly dictates the behavior of the side population of high-grade GSCs $[9,34]$. These exciting observations prompted recent discovery efforts to identify potential modulators of ABCG2 [35]. As the etiologic mechanisms responsible for ABCG1-mediated suppression of ER stress and glioma survival become elucidated [36-41], future therapeutic strategies designed to blocking these adaptations may improve treatment efficacy for this deadly brain malignancy.
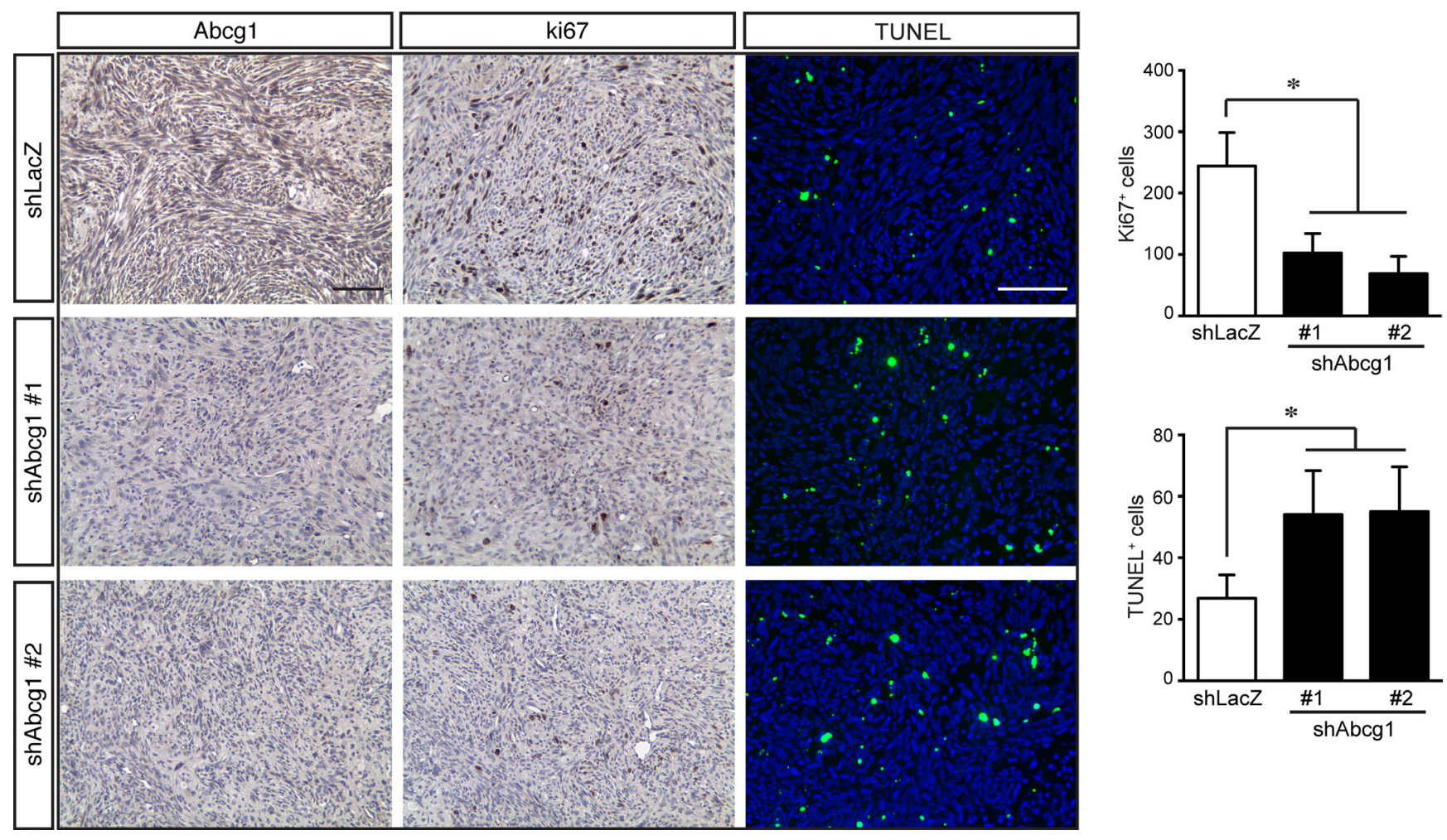

Figure 5: Abcg1 knockdown increases glioblastoma apoptosis in vivo. Glioma sections from control mice (shLacZ) reveal reduced Abcg1 immunostaining relative to mice harboring tumors with sh $\mathrm{Abcg} 1$ knockdown. Following Abcg1 knockdown, there was decreased glioma growth $\left(\% \mathrm{Ki} 67^{+}\right.$cells) and increased apoptosis $\left(\% \mathrm{TUNEL}{ }^{+}\right.$cells $)$. Error bars denote mean $\pm \mathrm{SD} .\left(^{*}\right) p<0.05$. 


\section{MATERIALS AND METHODS}

\section{Cell lines and mice}

Mouse malignant glioma NPcis cell lines, K1861 and $\mathrm{K} 4622$, were derived from C57BL/6J $\operatorname{Trp} 53^{+/ /} / \mathrm{Nf}^{+/-}$ mice (Dr. Karlyne Reilly, National Cancer Institute) [22]. These lines were maintained at $37^{\circ} \mathrm{C}$ in $5 \% \mathrm{CO}_{2}$ in Dulbecco's modified Eagle medium containing 10\% fetal bovine serum. All mouse procedures were performed in accordance with an approved Animal Studies protocol at Washington University.

\section{Lentivirus infections}

Prior to the lentiviral shAbcgl infection, NPcis cells were transduced with lentiviral FUW-GL vector (Dr. Joshua Rubin) for bioluminescence imaging. Abcgl shRNA or control shLacZ-containing pLKO.1 plasmid was co-transfected with $\mathrm{pMDLg} / \mathrm{pRRE}$, pRSV-REV and pCMV-VSV-G into HEK293T cells using the FuGENE HD transfection reagent (Roche). NPcis cells were dissociated into single cells by trypsin and transduced with viral supernatants from HEK293T cells with polybrene $(8 \mu \mathrm{g} / \mathrm{mL})$ for 48 hours. The knockdown efficiency of the $A b c g 1$ shRNA constructs was evaluated by Western blotting. Lentiviral constructs used are listed in Table S1.

\section{Western blotting}

Western blotting was performed as previously reported [42] using the primary antibodies listed in Table S2.

\section{Intracranial injections}

Injections were performed as previously described [43]. 3 to 5-week-old male wild-type C57BL/6J purchased from Taconic were used. NPcis cells were prepared in a $2.5 \times 10^{4}$ cell $/ \mu \mathrm{L}$ PBS solution and $2 \mu \mathrm{L}\left(5 \times 10^{4}\right)$ cells were sterilely implanted intracranially into the right side striatum using a stereotactic device (coordinates $=$ posterior $3 \mathrm{~mm}$ from the bregma, lateral $1.5 \mathrm{~mm}$ (right), and depth 2.5 from dura mater). Mice were monitored and euthanized after neurological symptoms developed.

\section{In vitro cell proliferation and death}

NPcis cells were trypsinized and plated onto $50 \mu \mathrm{g} /$ $\mathrm{mL}$ poly-D-lysine-coated and $10 \mu \mathrm{g} / \mathrm{mL}$ fibronectin-coated 24-well plates in defined culture medium. After 24 hours, cells were fixed in $4 \%$ paraformaldehyde. Proliferating and apoptotic cells were detected using antibodies to Ki67 and cleaved caspase- 3 , respectively. The percent of Ki67 $7^{+}$ or cleaved caspase $-3^{+}$cells were determined as a percent of the total cell number (DAPI ${ }^{+}$cells).

\section{Human specimens and immunostaining}

The use of human subject materials was approved by the institutional review board of the Washington University School of Medicine. Sixteen intracranial glioblastomas (WHO grade IV) and seven surgically obtained brain control cases were identified. Corresponding formalinfixed paraffin-embedded blocks from the pathology archives were used for immunohistochemistry. Paraffin sections were processed [44] prior to staining with appropriate antibodies (Table S2). The TUNEL labeling was performed using a fluorescence-based in situ cell death detection kit (Roche Diagnostics). The numbers of $\mathrm{Ki}^{+} 7^{+}$and TUNEL-positive cells were counted within the tumors per surface area $\left(0.1 \mathrm{~mm}^{2}\right)$.

\section{Bioluminescence imaging}

Animals were given $150 \mu \mathrm{g} / \mathrm{mL}$ D-luciferin (Gold Biotech) in PBS through intraperitoneal injection and imaged with a charge-coupled device (CCD) camerabased bioluminescence imaging system (IVIS50; PerkinElmer, Hopkinton, MA) with exposure time 10 secs 1 minute, binning 8 , field of view 12 , f/stop 1 , open filter. Signal was displayed as photons $/ \mathrm{sec} / \mathrm{cm}^{2} / \mathrm{sr}$ [45].

\section{Survival outcome analyses}

The survival outcome analyses were performed on the GSE16011 dataset using the R package "RMS". Each GBM sample (159 out of 276 total samples) was assigned to a TCGA subtype (Classical, Mesenchymal, Neural, or Proneural) based on the 10-nearest neighbors algorithm as previously described [46]. Kaplan-Meier survival plots were generated, along with log rank tests, to compare survival differences between the low/highexpression groups. Multivariate Cox models were fitted to gene expression (binary format, as dichotomized by the median) with incorporation of classic clinicopathological parameters (e.g., TCGA molecular subtype, age and KPS) to assess the independent prognostic ability of the candidate gene by the adjusted hazard ratio and associated 95\% confidence interval (CI). Age and KPS were incorporated in the format of restricted cubic spline basis function with three knots in order to relax the linearity assumption.

\section{Statistical analysis}

Each experiment was performed with samples from at least three independent groups. All data were processed and graphed in Prism GraphPad 6.0 with descriptive statistics calculated. The difference between experimental groups was assessed by one-way ANOVA. Survival curves were generated by the Kaplan-Meier method, and the Log-rank (Mantel-Cox) test was used to compare 
between/among treatments. All tests were two-sided with a significance level of $5 \%$ unless otherwise noted.

\section{ACKNOWLEDGMENTS}

We thank the Broad Institute RNAi Consortium (TRC), the Children's Discovery Institute (CDI), the Genome Institute at Washington University (TGI), and the Molecular Imaging Center at Washington University.

\section{GRANT SUPPORT}

This work was partly funded by a grant from the National Institutes of Health (R01-NS065547 to D.H.G.), while core services were partly defrayed by funding from the National Institutes of Health (P50-CA094056). Y.-H. Chen was the recipient of a fellowship from the American Brain Tumor Association supported by the Emily Dorfman Foundation for Children in memory of Emily Ann Dorfman.

\section{CONFLICTS OF INTEREST}

None to disclose.

\section{REFERENCES}

1. Kesari S, Schiff D, Henson JW, Muzikansky A, Gigas DC, Doherty L, Batchelor TT, Longtine JA, Ligon KL, Weaver S, Laforme A, Ramakrishna N, Black PM, et al. Phase II study of temozolomide, thalidomide, and celecoxib for newly diagnosed glioblastoma in adults. Neuro Oncol. 2008; 10:300-308.

2. Werner MH, Phuphanich S, Lyman GH. The increasing incidence of malignant gliomas and primary central nervous system lymphoma in the elderly. Cancer. 1995; 76:1634-1642.

3. Baumann F, Bjeljac M, Kollias SS, Baumert BG, Brandner S, Rousson V, Yonekawa Y, Bernays RL. Combined thalidomide and temozolomide treatment in patients with glioblastoma multiforme. J Neurooncol. 2004; 67:191-200.

4. Balasubramaniyan V, Vaillant B, Wang S, Gumin J, Butalid ME, Sai K, Mukheef F, Kim SH, Boddeke HW, Lang F, Aldape K, Sulman EP, Bhat KP, et al. Aberrant mesenchymal differentiation of glioma stem-like cells: implications for therapeutic targeting. Oncotarget. 2015; 6:31007-17. doi: 10.18632/oncotarget.5219.

5. Daniele S, Costa B, Zappelli E, Da Pozzo E, Sestito S, Nesi G, Campiglia P, Marinelli L, Novellino E, Rapposelli S, Martini C. Combined inhibition of AKT/mTOR and MDM2 enhances Glioblastoma Multiforme cell apoptosis and differentiation of cancer stem cells. Sci Rep. 2015; 5:9956.

6. Singh SK, Hawkins C, Clarke ID, Squire JA, Bayani J, Hide T, Henkelman RM, Cusimano MD, Dirks PB. Identification of human brain tumour initiating cells. Nature. 2004; 432:396-401.

7. Singh SK, Clarke ID, Terasaki M, Bonn VE, Hawkins C, Squire J, Dirks PB. Identification of a cancer stem cell in human brain tumors. Cancer Res. 2003; 63:5821-5828.

8. Liu G, Yuan X, Zeng Z, Tunici P, Ng H, Abdulkadir IR, Lu L, Irvin D, Black KL, Yu JS. Analysis of gene expression and chemoresistance of CD133+ cancer stem cells in glioblastoma. Mol Cancer. 2006; 5:67.

9. Bleau AM, Hambardzumyan D, Ozawa T, Fomchenko EI, Huse JT, Brennan CW, Holland EC. PTEN/PI3K/Akt pathway regulates the side population phenotype and ABCG2 activity in glioma tumor stem-like cells. Cell Stem Cell. 2009; 4:226-235.

10. Bao S, Wu Q, McLendon RE, Hao Y, Shi Q, Hjelmeland AB, Dewhirst MW, Bigner DD, Rich JN. Glioma stem cells promote radioresistance by preferential activation of the DNA damage response. Nature. 2006; 444:756-760.

11. Golebiewska A, Bougnaud S, Stieber D, Brons NH, Vallar L, Hertel F, Klink B, Schrock E, Bjerkvig R, Niclou SP. Side population in human glioblastoma is non-tumorigenic and characterizes brain endothelial cells. Brain. 2013; 136:1462-1475.

12. Wang N, Lan D, Chen W, Matsuura F, Tall AR. ATP-binding cassette transporters G1 and G4 mediate cellular cholesterol efflux to high-density lipoproteins. Proc Natl Acad Sci USA 2004; 101:9774-9779.

13. Tarling EJ, Edwards PA. ATP binding cassette transporter G1 (ABCG1) is an intracellular sterol transporter. Proc Natl Acad Sci USA. 2011; 108:19719-19724.

14. Klucken J, Buchler C, Orso E, Kaminski WE, PorschOzcurumez M, Liebisch G, Kapinsky M, Diederich W, Drobnik W, Dean M, Allikmets R, Schmitz G. ABCG1 (ABC8), the human homolog of the Drosophila white gene, is a regulator of macrophage cholesterol and phospholipid transport. Proc Natl Acad Sci USA. 2000; 97:817-822.

15. Kennedy MA, Barrera GC, Nakamura K, Baldan A, Tarr P, Fishbein MC, Frank J, Francone OL, Edwards PA. ABCG1 has a critical role in mediating cholesterol efflux to HDL and preventing cellular lipid accumulation. Cell Metab. 2005; 1:121-131.

16. Chen YH, McGowan LD, Cimino PJ, Dahiya S, Leonard JR, Lee da Y, Gutmann DH. Mouse low-grade gliomas contain cancer stem cells with unique molecular and functional properties. Cell Rep. 2015; 10:1899-1912.

17. Verhaak RG, Hoadley KA, Purdom E, Wang V, Qi Y, Wilkerson MD, Miller CR, Ding L, Golub T, Mesirov JP, Alexe G, Lawrence M, O'Kelly M, et al. Integrated genomic analysis identifies clinically relevant subtypes of glioblastoma characterized by abnormalities in PDGFRA, IDH1, EGFR, and NF1. Cancer Cell. 2010; 17:98-110.

18. Reilly KM, Loisel DA, Bronson RT, McLaughlin ME, Jacks T. Nf1;Trp53 mutant mice develop glioblastoma with evidence of strain-specific effects. Nat Genetics. 2000; 26:109-113.

19. Gursel DB, Connell-Albert YS, Tuskan RG, Anastassiadis T, Walrath JC, Hawes JJ, Amlin-Van Schaick JC, Reilly KM. Control of proliferation in astrocytoma cells by the receptor tyrosine kinase/PI3K/AKT signaling axis and the use of PI- 
103 and TCN as potential anti-astrocytoma therapies. Neuro Oncol. 2011; 13:610-621.

20. Ozawa T, Riester M, Cheng YK, Huse JT, Squatrito M, Helmy K, Charles N, Michor F, Holland EC. Most human non-GCIMP glioblastoma subtypes evolve from a common proneural-like precursor glioma. Cancer Cell. 2014; 26:288-300.

21. Network CGAR. Comprehensive genomic characterization defines human glioblastoma genes and core pathways. Nature. 2008; 455:1061-1068.

22. Banerjee S, Byrd JN, Gianino SM, Harpstrite SE, Rodriguez FJ, Tuskan RG, Reilly KM, Piwnica-Worms DR, Gutmann DH. The neurofibromatosis type 1 tumor suppressor controls cell growth by regulating signal transducer and activator of transcription-3 activity in vitro and in vivo. Cancer Res. 2010; 70:1356-1366.

23. Zemp FJ, McKenzie BA, Lun X, Maxwell L, Reilly KM, McFadden G, Yong VW, Forsyth PA. Resistance to oncolytic myxoma virus therapy in $\mathrm{nf1}(-/-) / \operatorname{trp} 53(-/-)$ syngeneic mouse glioma models is independent of anti-viral type-I interferon. PloS One. 2013; 8:e65801.

24. Luo B, Lee AS. The critical roles of endoplasmic reticulum chaperones and unfolded protein response in tumorigenesis and anticancer therapies. Oncogene. 2013; 32:805-818.

25. Li J, Lee AS. Stress induction of GRP78/BiP and its role in cancer. Curr Mol Med. 2006; 6:45-54.

26. Bajenaru ML, Hernandez MR, Perry A, Zhu Y, Parada LF, Garbow JR, Gutmann DH. Optic nerve glioma in mice requires astrocyte $\mathrm{Nf1}$ gene inactivation and $\mathrm{Nf1}$ brain heterozygosity. Cancer Res. 2003; 63:8573-8577.

27. Aleidi SM, Howe V, Sharpe LJ, Yang A, Rao G, Brown AJ, Gelissen IC. The E3-ubiquitin ligases, HUWE1 and NEDD41 , are involved in the post-translational regulation of the ABCG1 and ABCG4 lipid transporters. J Biol Chem. 2015.

28. Zhang H, Nie W, Zhang X, Zhang G, Li Z, Wu H, Shi Q, Chen Y, Ding Z, Zhou X, Yu R. NEDD4-1 regulates migration and invasion of glioma cells through CNrasGEF ubiquitination in vitro. PloS One. 2013; 8:e82789.

29. Dai B, Pieper RO, Li D, Wei P, Liu M, Woo SY, Aldape KD, Sawaya R, Xie K, Huang S. FoxM1B regulates NEDD4-1 expression, leading to cellular transformation and full malignant phenotype in immortalized human astrocytes. Cancer Res. 2010; 70:2951-2961.

30. Zhang P, de Gooijer MC, Buil LC, Beijnen JH, Li G, van Tellingen $\mathrm{O}$. $\mathrm{ABCB} 1$ and $\mathrm{ABCG} 2$ restrict the brain penetration of a panel of novel EZH2-Inhibitors. Int $\mathrm{J}$ Cancer. 2015; 137:2007-2018.

31. Lin F, de Gooijer MC, Roig EM, Buil LC, Christner SM, Beumer JH, Wurdinger T, Beijnen JH, van Tellingen $\mathrm{O}$. $\mathrm{ABCB} 1, \mathrm{ABCG} 2$, and PTEN determine the response of glioblastoma to temozolomide and ABT-888 therapy. Clin Cancer Res. 2014; 20:2703-2713.

32. Shi L, Wang Z, Sun G, Wan Y, Guo J, Fu X. miR-145 inhibits migration and invasion of glioma stem cells by targeting ABCG2. Neuromolecular Med. 2014; 16:517-528.
33. Gong W, Wang Z, Wan Y, Shi L, Zhou Y. Downregulation of ABCG2 protein inhibits migration and invasion in U251 glioma stem cells. Neuroreport. 2014; 25:625-632.

34. Bleau AM, Huse JT, Holland EC. The ABCG2 resistance network of glioblastoma. Cell Cycle. 2009; 8:2936-2944.

35. Mittapalli RK, Chung AH, Parrish KE, Crabtree D, Halvorson $\mathrm{KG}, \mathrm{Hu}$ G, Elmquist WF, Becher OJ. ABCG2 and $\mathrm{ABCB} 1$ limit the efficacy of dasatinib in a PDGF-B driven brainstem glioma model. Mol Cancer Ther. 2016.

36. Suzuki K, Gerelchuluun A, Hong Z, Sun L, Zenkoh J, Moritake T, Tsuboi K. Celecoxib enhances radiosensitivity of hypoxic glioblastoma cells through endoplasmic reticulum stress. Neuro Oncol. 2013; 15:1186-1199.

37. Liu AJ, Wang SH, Chen KC, Kuei HP, Shih YL, Hou SY, Chiu WT, Hsiao SH, Shih CM. Evodiamine, a plant alkaloid, induces calcium/JNK-mediated autophagy and calcium/mitochondria-mediated apoptosis in human glioblastoma cells. Chem Biol Interact. 2013; 205:20-28.

38. Johnson DR, Galanis E. Medical management of high-grade astrocytoma: current and emerging therapies. Semin Oncol. 2014; 41:511-522.

39. Ciechomska IA, Gabrusiewicz K, Szczepankiewicz AA, Kaminska B. Endoplasmic reticulum stress triggers autophagy in malignant glioma cells undergoing cyclosporine a-induced cell death. Oncogene. 2013; 32:1518-1529.

40. Cho HY, Wang W, Jhaveri N, Lee DJ, Sharma N, Dubeau L, Schonthal AH, Hofman FM, Chen TC. NEO212, temozolomide conjugated to perillyl alcohol, is a novel drug for effective treatment of a broad range of temozolomideresistant gliomas. Mol Cancer Ther. 2014; 13:2004-2017.

41. Booth L, Roberts JL, Cruickshanks N, Grant S, Poklepovic A, Dent P. Regulation of OSU-03012 toxicity by ER stress proteins, ER stress-inducing drugs. Mol Cancer Ther. 2014; 13:2384-2398.

42. Lee da Y, Yeh TH, Emnett RJ, White CR, Gutmann DH. Neurofibromatosis-1 regulates neuroglial progenitor proliferation and glial differentiation in a brain regionspecific manner. Genes Dev. 2010; 24:2317-2329.

43. Kaul A, Chen YH, Emnett RJ, Dahiya S, Gutmann DH. Pediatric glioma-associated KIAA1549:BRAF expression regulates neuroglial cell growth in a cell type-specific and mTOR-dependent manner. Genes Dev. 2012; 26:2561-2566.

44. Dasgupta B, Gutmann DH. Neurofibromin regulates neural stem cell proliferation, survival, and astroglial differentiation in vitro and in vivo. J Neurosci. 2005; 25:5584-5594.

45. Gross S, Piwnica-Worms D. Real-time imaging of ligandinduced IKK activation in intact cells and in living mice. Nat Methods. 2005; 2:607-614.

46. Pong WW, Walker J, Wylie T, Magrini V, Luo J, Emnett RJ, Choi J, Cooper ML, Griffith M, Griffith OL, Rubin JB, Fuller GN, Piwnica-Worms D, et al. F11R is a novel monocyte prognostic biomarker for malignant glioma. PloS One. 2013; 8:e 77571. 